\title{
Closed-Form ADOP Expressions for Single-Frequency GNSS-Based Attitude Determination
}

\author{
D. Odijk, P.J.G. Teunissen, A.R. Amiri-Simkooei \\ Delft Institute of Earth Observation and Space Systems (DEOS), Delft University of Technology, \\ Kluyverweg 1, 2629 HS Delft, The Netherlands, e-mail: D.Odijk@TUDelft.nl
}

\begin{abstract}
Integer ambiguity resolution is a prerequisite to high-precision real-time GNSS-based attitude determination. The ADOP is a well-known scalar measure to infer whether ambiguity resolution can be expected successful or not. To compute ADOP it is sufficient to have knowledge about the measurement setup and the measurements noise characteristics; hence it can be used as a planning tool. In this contribution we present closed-form expressions for the ADOP in case of attitude determination. Using these expressions one may infer the impact of GNSS design aspects such as number of satellites, choice of frequency and the precision of the phase and code observables. In addition, they are useful to quantify the influence of the number of antennas in the configuration and the use of geometric constraints, such as the lengths of the baselines and/or the angles between the baselines in the configuration. In this article the behavior of the ADOPs as function of these design aspects will be evaluated for several GPS attitude determination scenarios.
\end{abstract}

Keywords. GNSS, attitude determination, baseline constraints, LAMBDA method, ADOP

\section{Introduction}

Crucial to GNSS-based attitude determination of vehicles or platforms is the resolution of the integer ambiguities of the relative carrier-phase observations. A widely used approach for this is the integer least-squares search as implemented in the LAMBDA method, see e.g. Han et al. (1997), Park and Teunissen (2003), Dai et al. (2004) and Li et al. (2005). For successful ambiguity resolution it is required that the probability that the estimated integer solution coincides with the correct integers (the ambiguity success rate) is sufficiently high. One way to get insight into this is to evaluate the ADOP (Ambiguity Dilution Of Precision). This ADOP measure is derived from the variance matrix of the float ambiguity solution and is thus purely based on the assumptions in the mathematical model underlying.
In Teunissen and Odijk (1997) closed-form expressions were derived for the ADOP in a range of GPS positioning scenarios. These ADOP expressions are then a function of the model assumptions, such as number of satellites and receivers, number of frequencies and epochs, and the assumptions concerning the stochastic properties of the observations.

In this article we will present closed-form expressions for the ADOP in case of attitude determination. Hereby we take the following restrictions into account. First, we only discuss instantaneous attitude determination, based on a single epoch of singlefrequency phase and code (pseudo-range) data. We only present expressions for two- or three-antenna GNSS attitude determination systems. Although these antennas are usually connected to a common oscillator, implying that there are no receiver clock errors, in this article however receiver clock errors are taking into account because of the presence of unknown line biases, i.e. differential errors caused by differences in cable lengths between the antennas and oscillator. These line biases show up as receiver clock errors and as a consequence the ambiguities to be estimated are double-differenced (DD). It is finally assumed that atmospheric errors do not play a role since the distances between the antennas are very short, and that errors due to multipath are absent.

Using the expressions presented in this article one will also be able to analyze the influence of geometric constraints on ADOP in case of attitude determination. These constraints on the baseline lengths and/or angle between baselines will be treated as stochastic constraints, to serve two goals: (i) in case of a rigid antenna platform these constraints can be applied 'hard' by setting the standard deviations of the constraints to zero, (ii) in case of more flexible platforms (e.g. in airplanes or ships, when the baselines can be longer, e.g. a few $\mathrm{m}$ ) the constraints can be applied more loosely by setting their standard deviations to certain appropriate values.

The paper is set up as follows. In Sect. 2 the ADOP concept is reviewed. Sect. 3 reviews the ADOP expressions in case of positioning, while Sect. 4 presents the expressions for attitude determination. 
Examples are given in Sect. 5 and finally in Sect. 6 the conclusion follows. We remark that the results in this paper are given without proof. For proofs we refer to Odijk and Teunissen (2008).

\section{Ambiguity Dilution of Precision}

GNSS models for fast and precise relative applications can all be cast into the following model of linear(ized) observation equations:

$$
E\{y\}=A a+B b, \quad D\{y\}=Q_{y}
$$

where $E\{\cdot\}$ denotes the expectation operator and $D\{\cdot\}$ the dispersion operator. The vector $y$ denotes the normally distributed GNSS data vector ('observed-minus-computed' in case of a linearized model), whereas vectors $a$ (of order $n$ ) and $b$ (of order $o$ ) denote the unknown parameter vectors, for which $A$ and $B$ are the corresponding design matrices. Note that $a$ contains the integer carrierphase ambiguities, $a \in \mathbb{Z}^{n}$, and $b$ the remaining (real-valued) parameters, $b \in \mathbb{R}^{o}$. The stochastic properties of the observations are included in $Q_{y}$, the variance matrix.

The procedure to solve the model in equation (1) is usually divided into three steps. In the first step we disregard the integer constraints on the ambiguities and perform a standard least-squares adjustment. As a result, we obtain the (real-valued) estimates of $a$ and $b$, together with their variance-covariance matrix:

$$
\left[\begin{array}{c}
\hat{a} \\
\hat{b}
\end{array}\right], \quad\left[\begin{array}{cc}
Q_{\hat{a}} & Q_{\hat{a} \hat{b}} \\
Q_{\hat{b} \hat{a}} & Q_{\hat{b}}
\end{array}\right]
$$

This solution is referred to as the 'float' solution. In the second step, the float ambiguity estimate $\hat{a}$ is used to compute the corresponding integer ambiguity estimate, denoted as $\check{a}$ :

$$
\check{a}=F(\hat{a})
$$

with $F: \mathbb{R}^{n} \mapsto \mathbb{Z}^{n}$, a mapping from the real to the integer space. Once the integer ambiguities are computed, they are used in a third step to correct the float estimate of the real-valued parameters $b$. As a result we obtain the 'fixed' solution:

$$
\check{b}=\hat{b} \mid \check{a}=\hat{b}-Q_{\hat{b} \hat{a}} Q_{\hat{a}}^{-1}(\hat{a}-\check{a})
$$

If the ambiguity success rate, i.e. the probability that the estimated integers coincide with the true ambiguities, is sufficiently close to one, the precision of the fixed solution can be described by the following variance matrix (in which the integer ambiguities are assumed non-stochastic):

$$
Q_{\breve{b}} \simeq Q_{\hat{b} \mid a}=Q_{\hat{b}}-Q_{\hat{b} \hat{a}} Q_{\hat{a}}^{-1} Q_{\hat{a} \hat{b}}
$$

The success of ambiguity resolution depends on the quality of the float ambiguity estimates: the more precise the float ambiguities, the higher the probability of estimating the correct integer ambiguities. A simple measure to infer the float ambiguity precision is the Ambiguity Dilution of Precision (ADOP) defined as (Teunissen, 1997):

$$
\mathrm{ADOP}=\left|Q_{\hat{a}}\right|^{\frac{1}{2 n}} \quad[\mathrm{cyc}]
$$

Advantage of this scalar measure is that by taking the determinant we capture information not only on the variances but also on the covariances between the ambiguities. By raising the determinant to the power $1 /(2 n)$, the scalar is, like the ambiguities themselves, expressed in cycles.

The ADOP is linked to the ambiguity success rate as follows (Teunissen, 1998):

$$
P_{\mathrm{ADOP}}=\left[2 \Phi\left(\frac{1}{2 \mathrm{ADOP}}\right)-1\right]^{n}
$$

with $\Phi(x)=\int_{-\infty}^{x} \frac{1}{\sqrt{2 \pi}} \exp \left\{-\frac{1}{2} v^{2}\right\} \mathrm{d} v$. Although this ADOP-based probability $P_{A D O P}$ is an approximation of the true success rate of integer leastsquares, in Verhagen (2005) it was by means of simulations demonstrated that they agree reasonably well.

Figure 1 shows $P_{\mathrm{ADOP}}$ as function of ADOP for varying levels of $n(n=1, \ldots, 20)$. It can be seen that the ADOP-based success rate decreases for increasing ADOP and this decrease is steeper the more ambiguities are involved. In general, Figure 1 shows that if ADOP is larger than about $0.12 \mathrm{cyc}$ $P_{\text {ADOP }}$ becomes significantly smaller than 1 .

As mentioned in the introduction, in case of attitude determination it is common to include constraints on (some of) the parameters. The model incorporating stochastic constraints on the realvalued parameters, denoted as $c$, reads:

$$
E\left\{\left[\begin{array}{l}
y \\
c
\end{array}\right]\right\}=\left[\begin{array}{ll}
A & B \\
0 & C
\end{array}\right]\left[\begin{array}{l}
a \\
b
\end{array}\right]
$$




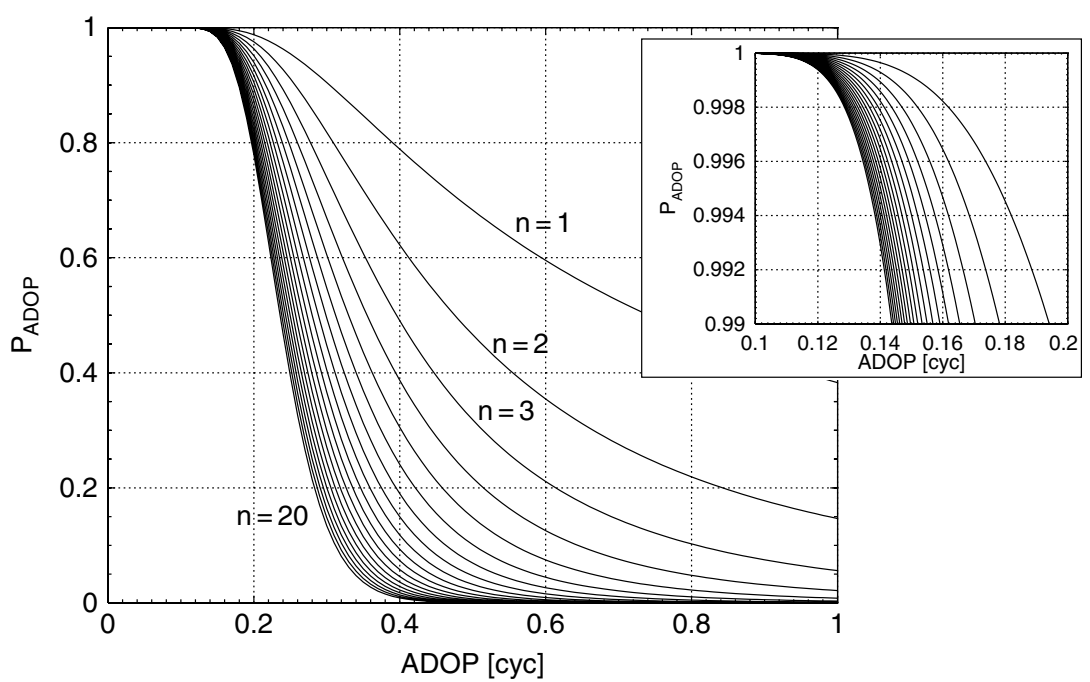

Fig. 1. $P_{\mathrm{ADOP}}$ versus $\mathrm{ADOP}$ for varying $n$.

The stochastic model, extended for the variance matrix of the constraints (denoted as $Q_{c}$ ), reads:

$$
D\left\{\left[\begin{array}{l}
y \\
c
\end{array}\right]\right\}=\left[\begin{array}{cc}
Q_{y} & 0 \\
0 & Q_{c}
\end{array}\right]
$$

The ADOP of the model in presence of constraints then follows as, see Odijk and Teunissen (2008):

$$
\operatorname{ADOP}=\operatorname{ADOP}(\infty)\left[\frac{\left|Q_{c}+Q_{\check{c}}(\infty)\right|}{\left|Q_{c}+Q_{\hat{c}}(\infty)\right|}\right]^{\frac{1}{2 n}}
$$

with $\operatorname{ADOP}(\infty)$ the $\mathrm{ADOP}$ of the model without constraints. Moreover, the float and fixed variance matrices of the constrained parameters are computed as $Q_{\hat{c}}(\infty)=C Q_{\hat{b}}(\infty) C^{T}$ and $Q_{\check{c}}(\infty)=C Q_{\breve{b}}(\infty) C^{T}$, respectively, where $Q_{\hat{b}}(\infty)$ and $Q_{\breve{b}}(\infty)$ are the float and fixed variance matrices of the real-valued parameters in absence of constraints. It is thus shown that in presence of constraints the ADOP can be directly computed from the ADOP in absence of constraints. It can be proved that the ratio in equation (10) as raised to the power $1 /(2 n)$ is always smaller than or equal to one. Consequently, $\mathrm{ADOP} \leq \operatorname{ADOP}(\infty)$. This is understandable, since addition of constraints makes the model stronger. In the limiting case, if $Q_{c}=0$, the constraints maximally contribute to the ambiguity precision; they have become hard constraints. On the other hand, if $Q_{c}=\infty$, the constraints do not have any weight and do not contribute at all to the ambiguity precision; i.e. $\mathrm{ADOP}=\operatorname{ADOP}(\infty)$.

\section{Positioning ADOP}

In this section the closed-form ADOP expressions for GNSS-based positioning are reviewed.

Let us first consider a single baseline, i.e. two GNSS antennas tracking $m$ satellites. In that case, there are $n=m-1$ DD ambiguities. The closedform expression for the single-frequency singleepoch ADOP was derived in Teunissen (1997) as

$$
\operatorname{ADOP}_{r=2}^{\mathrm{pos}}=m^{\frac{1}{2(m-1)}} \frac{\sigma_{\phi}}{\lambda}\left(1+\frac{\sigma_{p}^{2}}{\sigma_{\phi}^{2}}\right)^{\frac{3}{2(m-1)}}
$$

with $m$ the number of satellites tracked, $\lambda$ the wavelength, $\sigma_{\phi}$ the standard deviation of the singledifferenced phase observables and $\sigma_{p}$ the standard deviation of the single-differenced code observables. Usually, in case of GPS, $\sigma_{p}^{2} / \sigma_{\phi}^{2} \approx 10^{4}$, which implies that the term between the brackets in equation (11) is relatively large.

In case we have more than two - say $r$ - antennas simultaneously tracking the same $m$ satellites, then the ADOP for the $(r-1)(m-1)$ DD ambiguities $(r \geq 2)$ in the network can be related to the singlebaseline ADOP as (Teunissen and Odijk, 1997)

$$
\mathrm{ADOP}_{r}^{\mathrm{pos}}=\frac{1}{2} \sqrt{2} r^{\frac{1}{2(r-1)}} \mathrm{ADOP}_{r=2}^{\mathrm{pos}}
$$

It can be seen that for the purpose of ambiguity resolution the contribution of an additional receiver/antenna is low: when going from two to three antennas the ADOP of the network is only 0.93 times its single-baseline counterpart. 


\section{Attitude Determination ADOP}

The ADOP expressions for positioning turn out to play a role in the expressions for attitude determination. This relation will be revealed in the current section for attitude determination based on two and three antennas, respectively.

\subsection{Two Antennas}

In two-antenna attitude determination one tries to solve for pitch/elevation $\alpha$ and heading/azimuth/yaw $\gamma$ of the baseline, plus its length $l$, which are related to the local East-North-Up coordinates as, see Figure 2:

$$
\left[\begin{array}{c}
E_{12} \\
N_{12} \\
U_{12}
\end{array}\right]=\left[\begin{array}{c}
l \cos \alpha \sin \gamma \\
l \cos \alpha \cos \gamma \\
l \sin \alpha
\end{array}\right]
$$

In absence of any constraint, it can be shown that the ADOP in case of two-antenna attitude determination equals its single-baseline position counterpart (i.e. a reparametrization of the position vector does not affect ADOP). In presence of a constraint on the length of the baseline, having standard deviation $\sigma_{l}$, the ADOP can be easily obtained using equation (10), as

$$
\mathrm{ADOP}_{r=2}^{\mathrm{att}}=\operatorname{ADOP}_{r=2}^{\mathrm{pos}}\left[\frac{\sigma_{l}^{2}+\sigma_{l}^{2}(\infty)}{\sigma_{l}^{2}+\sigma_{l}^{2}(\infty)}\right]^{\frac{1}{2(m-1)}}
$$

with $\mathrm{ADOP}_{r=2}^{\mathrm{pos}}$ as in equation (11) and $\sigma_{\hat{l}}(\infty)$ and $\sigma_{\breve{l}}(\infty)$ the standard deviation of the float and fixed baseline lengths in absence of the constraint, respectively. To obtain $\sigma_{\hat{l}}^{2}(\infty)$ and $\sigma_{\breve{l}}^{2}(\infty)$ we need to linearize

$$
l(\infty)=\sqrt{E_{12}^{2}+N_{12}^{2}+U_{12}^{2}}
$$

i.e. the baseline length in absence of the constraint, completely determined by the East-North-Up

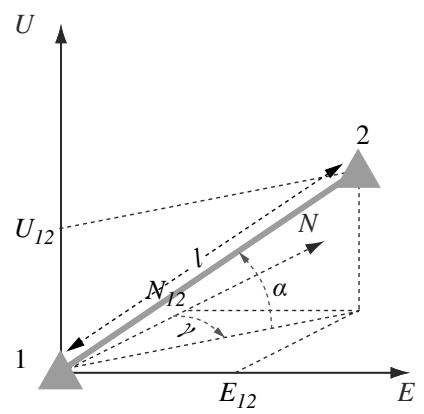

Fig. 2. Attitude determination based on a single baseline. coordinates. Application of the the variance propagation law results in:

$$
\sigma_{\hat{l}}^{2}(\infty)=\mu^{T} Q_{\hat{g}} \mu, \quad \sigma_{\check{l}}^{2}(\infty)=\mu^{T} Q_{\check{g}} \mu
$$

with $\mu=\frac{1}{l^{0}}\left(E_{12}^{0}, N_{12}^{0}, U_{12}^{0}\right)^{T}$ a unit vector based on the (approximated) position coordinates and $g=$ $\left(E_{12}, N_{12}, U_{12}\right)^{T}$ the position vector itself, with $Q_{\hat{g}}$ and $Q_{\breve{g}}$ its float and fixed variance matrices, respectively.

\subsection{Three Antennas}

If a third antenna is added to the system, such that the baseline between antennas 1 and 3 is non-collinear with the baseline between 1 and 2 , in addition to the pitch and heading also the roll angle $\beta$ can be determined (see Figure 3). With three antennas it becomes possible to determine the attitude by introducing a body frame (denoted using $u, v, w$ ) fixed to the vehicle, with the first antenna chosen as the origin of the body frame and the plane through the three antenna positions defining the $(u, v)$-plane. The $w$-axis is perpendicular to this plane. Both frames are connected as (for $i=1,2,3$ ):

$$
\left[\begin{array}{c}
E_{1 i} \\
N_{1 i} \\
U_{1 i}
\end{array}\right]=R_{w}(\gamma) R_{u}(-\alpha) R_{v}(\beta)\left[\begin{array}{c}
u_{i} \\
v_{i} \\
w_{i}
\end{array}\right]
$$

with $R_{u}, R_{v}$ and $R_{w}$ rotation matrices. The body coordinates of the antennas are $\left(u_{1}, v_{1}, w_{1}\right)=$ $(0,0,0),\left(u_{2}, v_{2}, w_{2}\right)=\left(0, l_{1}, 0\right)$ and $\left(u_{3}, v_{3}, w_{3}\right)=$ $\left(l_{2} \sin \varphi, l_{2} \cos \varphi, 0\right)$, with $l_{1}$ and $l_{2}$ the baseline lengths and $\varphi$ the angle between both baselines.

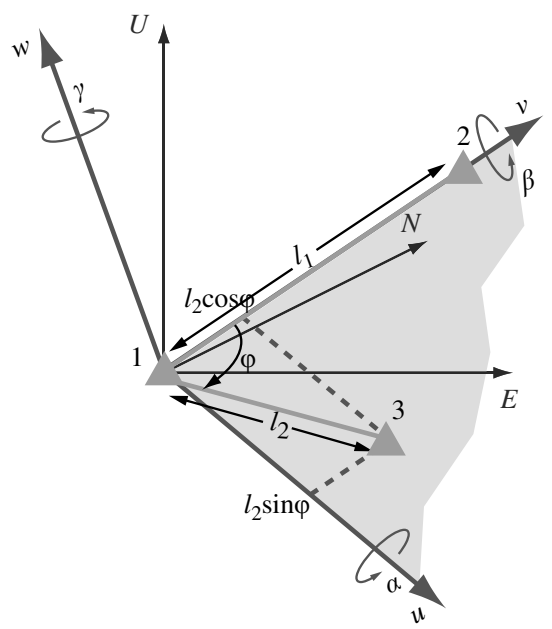

Fig. 3. Attitude determination based on three antennas. 
In absence of constraints on the baseline lengths and angle, there are now 6 unknowns: the three Euler angles $(\alpha, \beta$ and $\gamma)$, plus the two baseline lengths and baseline angle. Thus the 6 position coordinates are reparametrized into 6 other parameters. As in the dual-antenna case, it can be proved that the three-antenna attitude ADOP equals the threeantenna positioning ADOP, since in the latter case there are also 6 unknowns (coordinates). In presence of constraints on both baseline lengths, the ADOP becomes, using equation (10):

$$
\operatorname{ADOP}_{r=3}^{\text {att }}\left(\sigma_{\varphi}=\infty\right)=\operatorname{ADOP}_{r=3}^{\mathrm{pos}}\left[\frac{\left|Q_{l}+Q_{\grave{l}}(\infty)\right|}{\left|Q_{l}+Q_{\hat{l}}(\infty)\right|}\right]^{\frac{1}{4(m-1)}}
$$

with $Q_{l}$ the variance matrix of the two length constraints and $Q_{\hat{l}}(\infty)$ extracted from:

$$
\left[\begin{array}{cc}
Q_{\hat{l}}(\infty) & Q_{\hat{l} \hat{\varphi}}(\infty) \\
Q_{\hat{\varphi} \hat{l}}(\infty) & \sigma_{\hat{\varphi}}^{2}(\infty)
\end{array}\right]=\left[\begin{array}{cc}
\mu^{T} Q_{\hat{g}} \mu & \mu^{T} Q_{\hat{g}} \eta \\
\eta^{T} Q_{\hat{g}} \mu & \eta^{T} Q_{\hat{g}} \eta
\end{array}\right]
$$

(Note: $Q_{\breve{l}}(\infty)$ is computed analogously based on $\left.Q_{\check{g}}(\infty)\right)$. Here $\mu=\operatorname{blkdiag}\left(\mu_{1}, \mu_{2}\right)$ where $\mu_{1}$ and $\mu_{2}$ are unit vectors based on the (approximated) coordinates of antennas 2 and $3, g=$ $\left(E_{12}, N_{12}, U_{12}, E_{13}, N_{13}, U_{13}\right)^{T}$ and $\eta$ a $6 \times 1$-vector obtained when the following expression is linearized:

$$
\varphi(\infty)=\arccos \frac{E_{12} E_{13}+N_{12} N_{13}+U_{12} U_{13}}{l_{1} l_{2}}
$$

where use is made of the inner product between the two baseline vectors. Now assume - in addition to the baseline length constraints - also a constraint on the baseline angle, having a standard deviation $\sigma_{\varphi}$. Then the ADOP in presence of both types of constraints can be computed from the ADOP in presence of baseline length constraints only, see equation (18):

$$
\mathrm{ADOP}_{r=3}^{\mathrm{att}}=A D O P_{r=3}^{a t t}\left(\sigma_{\varphi}=\infty\right)\left[\frac{\sigma_{\varphi}^{2}+\sigma_{\tilde{\varphi}}^{2}\left(\sigma_{\varphi}=\infty\right)}{\sigma_{\varphi}^{2}+\sigma_{\hat{\varphi}}^{2}\left(\sigma_{\varphi}=\infty\right)}\right]^{\frac{1}{4(m-1)}}
$$

with $\sigma_{\hat{\varphi}}^{2}\left(\sigma_{\varphi}=\infty\right)$ computed as

$$
\begin{aligned}
& \sigma_{\hat{\varphi}}^{2}\left(\sigma_{\varphi}=\infty\right)=\sigma_{\hat{\varphi}}^{2}(\infty)- \\
& \quad Q_{\hat{\varphi} \hat{l}}(\infty)\left[Q_{l}+Q_{\hat{l}}(\infty)\right]^{-1} Q_{\hat{l} \hat{\varphi}}(\infty)
\end{aligned}
$$

The ambiguity-fixed $\sigma_{\tilde{\varphi}}^{2}\left(\sigma_{\varphi}=\infty\right)$ is computed analogously by considering the fixed variance matrices.

\section{Examples}

As an illustration we computed instantaneous ADOPs for GPS-based attitude determination, using the presented closed-form expressions. In these computations we used the receiver-satellite geometry of permanent GPS station Delft $\left(52.0^{\circ} \mathrm{N}, 4.4^{\circ} \mathrm{E}\right)$, the Netherlands, for 1 January 2003 (00-24h UTC; 30s sampling interval; cut-off elevation: $15 \mathrm{deg}$ ). Singlefrequency (L1) phase and code data were assumed having (single-differenced) standard deviations of $\sigma_{\phi}=\sqrt{2} \cdot 3 \mathrm{~mm}$ and $\sigma_{p}=\sqrt{2} \cdot 30 \mathrm{~cm}$.

In the dual-antenna case the computations have been conducted using $\alpha^{0}=\gamma^{0}=0$, and with this choice the a priori coordinates are $\left(E_{12}^{0}, N_{12}^{0}, U_{12}^{0}\right)=$ $\left(0, l^{0}, 0\right)$. Note that this choice only affects ADOP in presence of a (soft) baseline length constraint, through vector $\mu$ in equation (14). It was numerically verified that with other choices for $\alpha^{0}$ and $\gamma^{0}$ ADOP is hardly changed. Concerning the a priori baseline length $l^{0}$, this is set to $l^{0}=1 \mathrm{~m}$ in the computations, although this choice does not affect ADOP at all since it gets eliminated in vector $\mu$ (which equals $(0,1,0)^{T}$ in this case).

In the triple-antenna case the a priori Euler angles were set to $\alpha^{0}=\beta^{0}=\gamma^{0}=0$, which means that the body frame coincides with the local EastNorth-Up frame. It was numerically verified that this a priori attitude hardly affects ADOP. The two baseline lengths were chosen as $l_{1}^{0}=l_{2}^{0}=1 \mathrm{~m}$ and this choice only affects ADOP in case of a constraint on the baseline angle. It should be realized that since both $\sigma_{\hat{\varphi}}\left(\sigma_{\varphi}=\infty\right)$ and its fixed counterpart are inversely proportional to the baseline length (see equation (21)). This implies that the standard deviation of the baseline angle constraint is allowed to be larger in case the baseline lengths are shorter (for example if $\sigma_{\varphi}$ is set to $5 \mathrm{deg}$ in case the baseline lengths are $1 \mathrm{~m}$, then the same ADOP level is reached in case the baseline lengths are $0.1 \mathrm{~m}$, but with $\sigma_{\varphi}$ taken equal to $50 \mathrm{deg}$ !). The a priori baseline angle was in all computations set to $\varphi^{0}=90 \mathrm{deg}$, since it turned out that ADOP is hardly changed by other choices of this angle.

\subsection{Two Antennas}

In the dual-antenna case, we first computed the ADOPs and ADOP-based success rates in absence of a baseline length constraint $\left(\sigma_{l}=\infty\right)$. These ADOPs, corresponding to their counterparts in case of single-baseline positioning, are plotted for the day in Figure 4. It can be seen that the ADOP can be large; almost 3 cycle in case of 4 satellites. The 

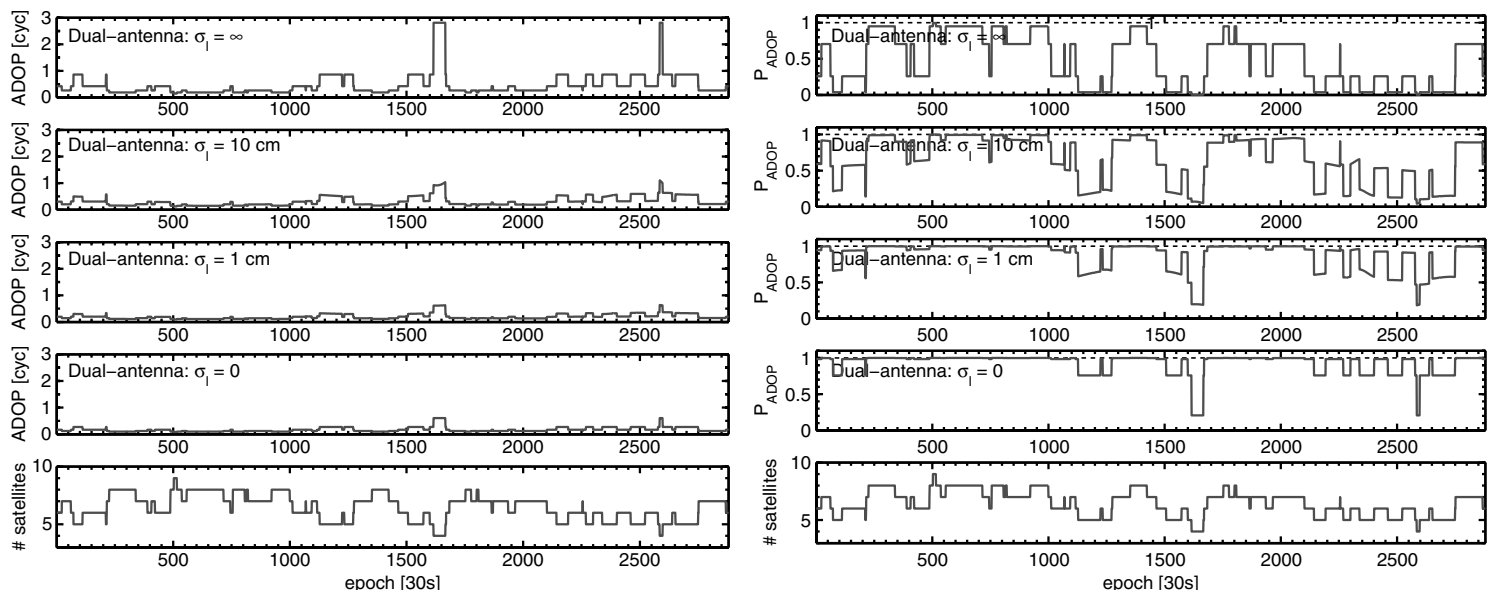

Fig. 4. Dual-antenna case. Shown are the ADOPs (left) and ADOP-based success rate $P_{\text {ADOP }}$ (right). The first row shows the results for $\sigma_{l}=\infty$ (no constraint); the second row the results for $\sigma_{l}=10 \mathrm{~cm}$; the third row those for $\sigma_{l}=1 \mathrm{~cm}$, while the fourth row shows the results for $\sigma_{l}=0$ (hard constraint). The last row gives the number of satellites during the day.

corresponding success rate is close to zero. Only with a large number of satellites, at least 9 , ADOP is sufficiently small such that the success rate approaches to 1 . Better results are obtained in case the baseline length is constrained. Using a standard deviation of $\sigma_{l}=10 \mathrm{~cm}$, though the ADOPs are smaller than without constraint, for many parts of the day the ADOP-based success rate is still insufficient. With a standard deviation of the constraint of $1 \mathrm{~cm}$ however, for many times (with at least 6-7 satellites) the ADOP is sufficiently small such that the success rate approaches to 1 . Even better results are obtained when the baseline length is incorporated as hard constraint $\left(\sigma_{l}=0\right)$.

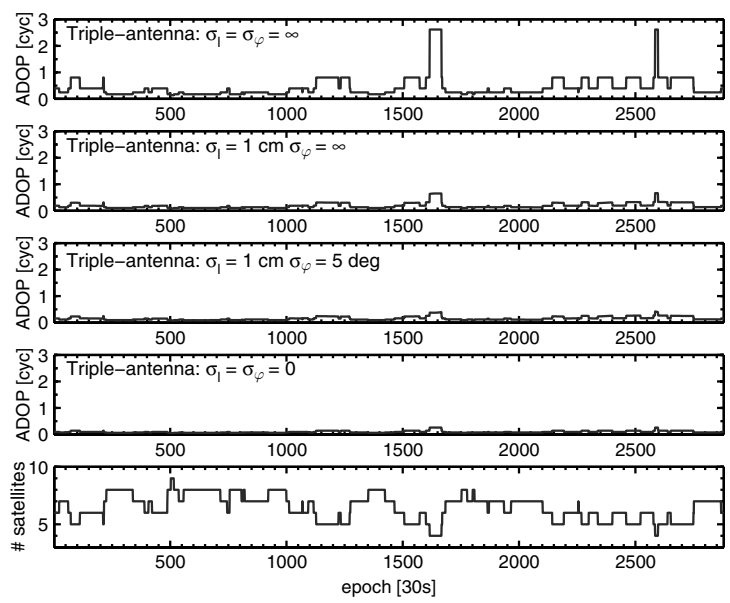

\subsection{Three Antennas}

In the triple-antenna case, the variance matrix of the two baseline lengths is assumed as a scaled identity matrix: $Q_{l}=\sigma_{l}^{2} I_{2}$. We first computed ADOPs in absence of any constraints $\left(\sigma_{l}=\sigma_{\varphi}=\infty\right)$, see Figure 5. As a result we obtain ADOPs that correspond to those of network-based positioning. Compared to the dual-antenna case, the ADOPs are decreased only marginally. Addition of two baseline length constraints with $\sigma_{l}=1 \mathrm{~cm}$ lower the ADOPs and hence increase the ADOP-based success rates significantly during the day. If we add a constraint on the angle between both baselines of $\sigma_{\varphi}=5 \mathrm{deg}$ as well, the success rates are close to 1 , except during
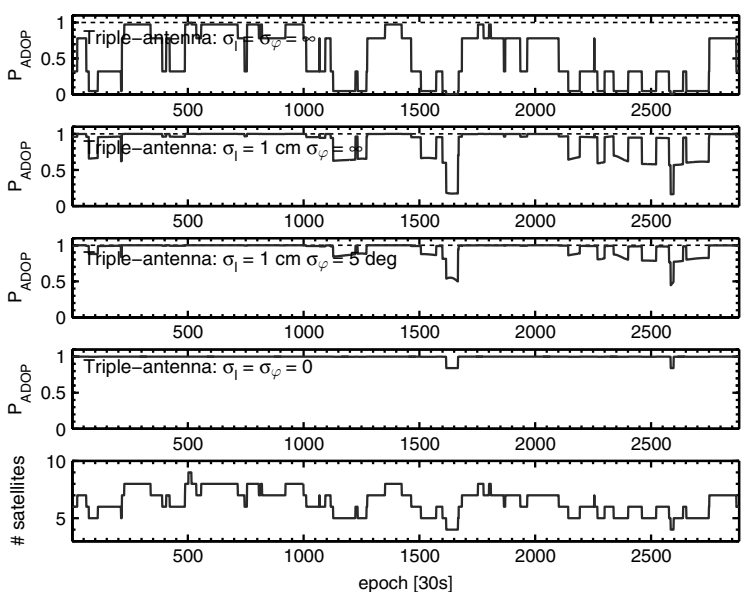

Fig. 5. Triple-antenna case. Shown are the ADOPs (left) and ADOP-based success rates $P_{\text {ADOP }}$ (right). The first row shows the results for $\sigma_{l}=\sigma_{\varphi}=\infty$ (no constraints); the second row those for $\sigma_{l}=1 \mathrm{~cm}$ and $\sigma_{\varphi}=\infty$, while the third row shows the results for $\sigma_{l}=1 \mathrm{~cm}$ and $\sigma_{\varphi}=5 \mathrm{deg}$. Finally, the fourth row shows the results using hard constraints: $\sigma_{l}=\sigma_{\varphi}=0$ and the last row gives the number of satellites during the day. 
periods with 5 satellites or less. The best results are of course obtained if the baseline lengths and angle may be hard constrained $\left(\sigma_{l}=\sigma_{\varphi}=0\right)$. In that case only during the (short) periods of having 4 satellites the ADOP-based success rate is not close to 1 . Note especially in this scenario the benefits of tripleantenna attitude determination: in the dual-antenna case still for some considerable time of the day the success rate is not sufficiently close to 1 , despite the hard constrained baseline length.

\section{Conclusion}

In this article closed-form expressions have been presented for GNSS-based attitude determination. It was shown that the ADOP of attitude determination corresponds to the ADOP of positioning in absence of constraints on baseline lengths and/or baseline angle. It was also demonstrated that the contribution of constraints to ADOP becomes immediately clear since they appear as additional (scaling) factors in the closed-form ADOP expressions.

\section{References}

Dai, L., K.V. Ling, and N. Nagarajan (2004). Real-time attitude determination for microsatellite by Lambda method combined with Kalman filtering. Proceedings of the 22nd AIAA ICSSC and Exhibit 2004, Monterey, CA, USA, May 9-12.

Han, S., K. Wong, and C. Rizos (1997). Instantaneous ambiguity resolution for real-time GPS attitude determination. Proceedings of the International Symposium on Kinematic Systems in Geodesy, Geomatics and Navigation, KIS97, Banff, Canada, June 3-6, 409-416.

Li, Y., K. Zhang, and R. Grenfell (2005). Improved Knight method based on narrowed search space for instantaneous attitude determination. Navigation, 52(2): 111-119.

Odijk, D., and P.J.G. Teunissen (2008). Ambiguity Dilution Of Precision: Diagnostic for Global Navigation Satellite System attitude determination. Submitted to Journal of Guidance, Control, and Dynamics.

Park, C., and P.J.G. Teunissen (2003). A new carrier phase ambiguity estimation for GNSS attitude determination systems. Proceedings of the International Symposium on GPS/GNSS, Tokyo, Japan, November 15-18.

Teunissen, P.J.G. (1997). A canonical theory for short GPS baselines. Part IV: Precision versus reliability. Journal of Geodesy, 71: 513-525.

Teunissen, P.J.G., and D. Odijk (1997). Ambiguity Dilution Of Precision: Definition, properties and application. Proceedings of ION GPS-1997, Kansas City, MO, USA, September 16-19, 891-899.

Teunissen, P.J.G. (1998). Success probability of integer GPS ambiguity rounding and bootstrapping. Journal of Geodesy, 72: 606-612.

Verhagen, S. (2005). On the reliability of integer ambiguity resolution. Navigation, 52(2): 99-110. 